\title{
Development and application of an AllGlo probe-based qPCR assay for detecting knockdown resistance $(k d r)$ mutations in Anopheles sinensis
}

Liang Bai ${ }^{1,2+}$, Guo-ding Zhu ${ }^{1,3+}{ }^{1}$, Hua-yun Zhou' ${ }^{1}$, Jian-xia Tang ${ }^{1}$, Ju-lin Li ${ }^{1}$, Sui Xu' ${ }^{1}$, Mei-hua Zhang ${ }^{1}$, Li-nong Yao ${ }^{4}$, Guang-quan Huang ${ }^{5}$, Yong-bin Wang ${ }^{6}$, Hong-wei Zhang ${ }^{7}$, Si-bao Wang ${ }^{2}$, Jun Cao ${ }^{1,8^{*}}$ and Qi Gao ${ }^{1,3^{*}}$

\begin{abstract}
Background: Anopheles sinensis is one of the most important malaria vectors in China and other Southeast Asian countries. High levels of resistance have been reported in this species due to the long-term use of insecticides, especially pyrethroids, for public health and agricultural purposes. Knockdown resistance $(k d r)$ caused by a single base pair mutation in the gene encoding the sodium channel is strongly associated with pyrethroid insecticide resistance in many Anopheles mosquitoes. There are few methods currently available for detecting kdr mutations in An. sinensis.

Methods: A novel AllGlo probe-based qPCR (AllGlo-qPCR) method was developed to screen for the predominant $k d r$ mutations in An. sinensis mosquitoes from the Jiangsu Province. The results from AllGlo-gPCR, allele-specific PCR (AS-PCR), and TaqMan-MGB probe-based GPCR (TaqMan-qPCR) were compared. A comparative analysis of the equipment required, ease of use and cost of the available methods was also performed. Finally, the AllGlo-qPCR method was used to detect the frequencies of $k d r$ mutations from the other four provinces in central China.

Results: Six kdr genotypes were detected in An. sinensis from the Jiangsu Province by DNA sequencing. The AllGlo-qPCR method detected all of the $k d r$ genotypes with a high level of accuracy ( $97 \%$ sensitivity and $98 \%$ specificity). AllGlo-qPCR correctly determined the $k d r$ genotypes of $98.73 \%$ of 158 An. sinensis samples, whereas TaqMan-qPCR and AS-PCR correctly identified $96.84 \%$ and $88.61 \%$ of mutations, respectively. Furthermore, the AllGlo-qPCR method is simpler to perform, requires less equipment, and exhibits a moderate expense cost comparing with the other tested methods of $k d r$ mutation detection. Samples collected from four of the other provinces in central China showed a high frequency of $k d r$ mutation in An. sinensis, as detected by the established AllGlo-qPCR method.

Conclusion: The novel AllGlo-qPCR method developed for $k d r$ mutation detection in An. sinensis exhibits greater specificity and sensitivity than currently available methods and is more cost-effective; therefore, it represents a useful tool for entomological surveillance.
\end{abstract}

Keywords: Anopheles sinensis, Knockdown resistance (kdr), AllGlo probe, qPCR

\footnotetext{
* Correspondence: caojuncn@hotmail.com; gaoqi54@hotmail.com

${ }^{\dagger}$ Equal contributors

${ }^{1}$ Key Laboratory of Parasitic Disease Control and Prevention (Ministry of

Health), and Jiangsu Provincial Key Laboratory of Parasite Molecular Biology,

Jiangsu Institute of Parasitic Diseases, Wuxi, Jiangsu Province, People's

Republic of China

${ }^{3}$ Department of Parasitology, Medical College of Soochow University,

Suzhou 215123, People's Republic of China

Full list of author information is available at the end of the article
} 


\section{Background}

Anopheles sinensis is an important member of the Anopheles hyrcanus group [1]. It is widely distributed in China, Korea, Japan and other Asian countries and is a major malaria vector, especially in central China [2]. There is increasing interest in this species due to its high abundance and modest susceptibility to malarial parasites $[3,4]$.

Vector control remains the primary component of malaria control strategies, even for countries that are in the malaria elimination phase [5]. Currently, the most accepted methods for treatment involve using the insecticide pyrethroid for indoor residual spraying (IRS) and insecticide-treated nets (ITNs) in the regions with a high risk of malarial transmission [6]. However, the large-scale use of pyrethroid for public health and agricultural purposes has resulted in the rapid spread of resistance in malaria vectors. Reports of pyrethroid resistance in $A n$. sinensis have increased dramatically in recent years $[7,8]$. Urgent action is needed to prevent the emergence of resistance at new sites and to maintain the effectiveness of vector control interventions in the short, medium and long-term. The latest global plan for insecticide resistance management in malaria vectors (GPIRM) released by the World Health Organization (WHO) advocates collecting baseline data associated with insecticide resistance in major vectors to strengthen scientific collaboration and to further understand the mechanisms of insecticide resistance at both global and national levels [9].

Pyrethroids continuously activate voltage-gated sodium channels (VGSCs) in insects, leading to spasms, paralysis and death. However, amino acid substitutions in segment 6 of domain II of the VGSC result in insensitivity to pyrethroids. Reduced sodium channel target-site sensitivity is a major mechanism of pyrethroid resistance and is referred to as knockdown resistance $(k d r)$ [10]. Many previous studies have demonstrated that the $k d r$ is strongly associated with insecticide resistance in Anopheles mosquitoes, including $A n$. sinensis $[7,11,12]$.

There are currently very few assays available to screen for DNA substitutions that lead to $k d r$ mutations in $A n$. sinensis. The most commonly used method is allelespecific PCR (AS-PCR) [7,13] (also known as competitive PCR amplification of a specific allele (cPASA) [11]), due to its relatively low cost. However, this technique can generate inaccurate results due to mismatches at the 3 ' end of the primer, and if the system is poorly optimized, it can be difficult to determine band brightness. To improve screening accuracy, a TaqMan-MGB probebased assay was developed to detect $k d r$ mutations [14]. Unfortunately, this approach is unable to identify all $k d r$ mutation genotypes within a single reaction: two parallel reaction tubes are required to provide complete mutation information. In this study, a novel AllGlo probebased screening method was developed to overcome these problems. This method is based on sequencing a portion of the sequence coding for VGSC in An. sinensis. Using this method, the frequencies of $k d r$ mutations in several provinces in central China were detected.

\section{Methods \\ Mosquito collection and identification}

Wild anopheline mosquitoes were collected from vector surveillance sites in Jiangsu Province in 2011 and were transferred to the insectary of the Key Laboratory on Technology for Parasitic Disease Control and Prevention, Ministry of Health, Jiangsu Institute of Parasitic Diseases (JIPD) in Wuxi, Jiangsu Province, China. Wild An. sinensis mosquitoes were also collected from the other provinces in central China, including Zhejiang, Henan, Shandong and Hubei (Figure 1). The wild mosquito species were identified by rDNA-ITS2 [15], and only those confirmed as An. sinensis were selected for the following studies. The laboratory colonies of $A n$. sinensis have been cultured in JIPD for more than thirty years without exposure to any insecticides.

\section{gDNA extraction}

Total genomic DNA was extracted from each individual mosquito using a Fast Tissue-to-PCR kit (Thermo scientific) according to the manufacturer's instructions. Briefly, one or two mosquito legs were added to a mixture of $100 \mu \mathrm{L}$ of Tissue Lysis Solution and $10 \mu \mathrm{L}$ of Proteinase K and were incubated at $55^{\circ} \mathrm{C}$ for $10 \mathrm{~min}$ and $95^{\circ} \mathrm{C}$ for $10 \mathrm{~min}$. Next, $100 \mu \mathrm{L}$ of Neutralization Solution $\mathrm{T}$ was added to the mixture, which was then centrifuged at $17,900 \times \mathrm{g}$ for $3 \mathrm{~min}$. The supernatant was transferred to a new tube and stored at $-30^{\circ} \mathrm{C}$ for species identification and $k d r$ detection.

\section{Nucleotide sequencing}

The $k d r$ genotypes of individual mosquitoes were determined by DNA sequencing. A partial segment of the VGSC gene that included the $k d r$ mutations was amplified from laboratory An. sinensis strains and randomly selected wild An. sinensis strains from the Jiangsu Province using the previously described primers $k d r-\mathrm{F}$ (5'-TGCCACTCCGTGT GTTTAGA-3') and $k d r$-R 5'-GAGCGATGATGATCC GAAAT-3') [7]. The PCR products were electrophoresed on a $1.5 \%$ agarose gel containing $0.5 \mu \mathrm{g} / \mathrm{mL}$ ethidium bromide. Direct PCR sequencing of both strands was performed by Genscript (Nanjing, China).

\section{Development of the AllGlo-qPCR method}

The nucleotide sequences generated as described above were aligned and compared to the available sequence in NCBI (GenBank NO: GI84646709), and the conserved region surrounding the $k d r$ site was selected for primer/probe design. We designed forward and reverse primers and 3 AllGlo probes based on the sequenced $k d r$ genotypes 


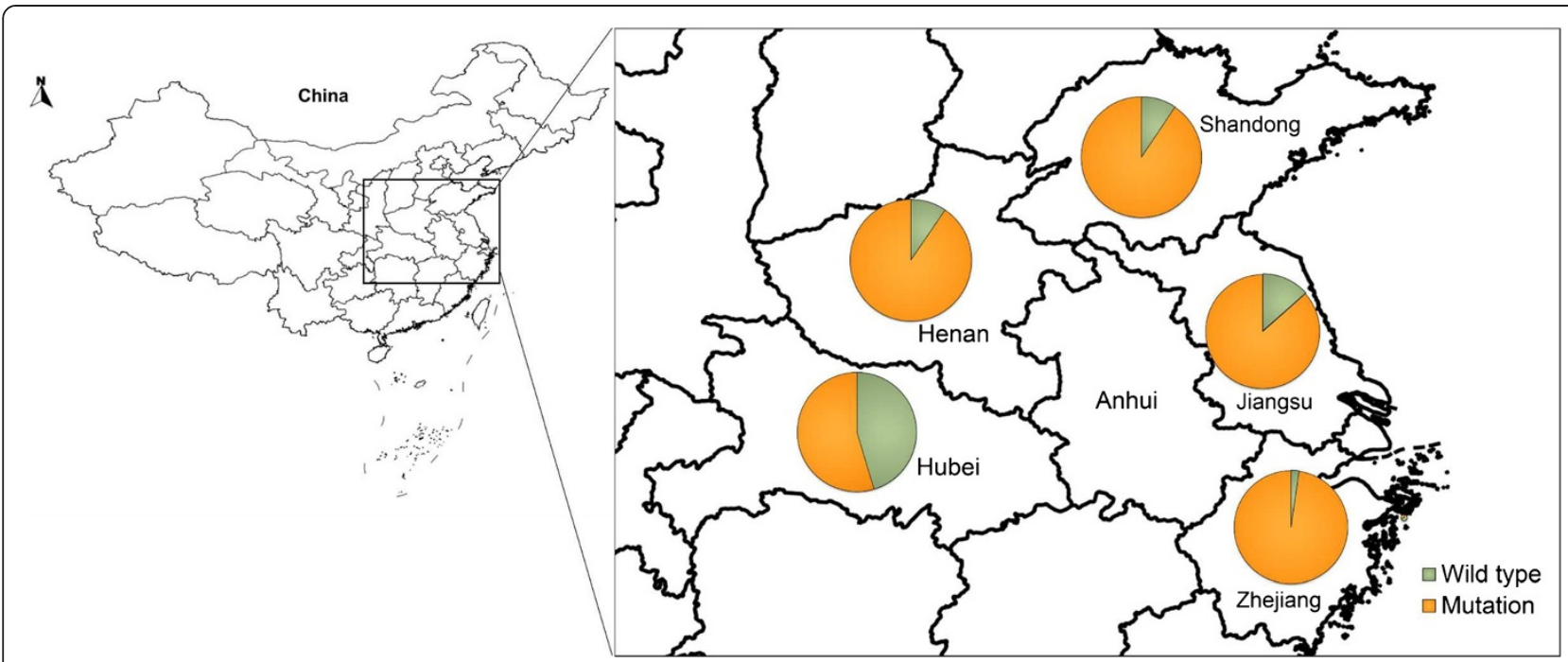

Figure 1 The collection sites for An. sinensis in central China.

(Shanghai Huirui Biotechnology Co., Ltd). The forward primer (primer-F: 5'-CCATTCTTCTTAGCCACTGTG3') and the reverse primer (primer-R: 5'-CTTATTA GAATCGGAGCAA-3') were standard oligonucleotides with no modifications. The $k d r$-TTG probe (MAR-TGA AACTTGGTGGTGAG-MAR) for detection of the wildtype allele was labelled with MAR at the $5^{\prime}$ end, the $k d r$ TTT probe (JUP-TGGAAACTTTGTGGTGA G-JUP) for detection of $k d r$-TTT was labelled with JUP and the $k d r$ TGT probe (NEP-TGAAACTGTGTGGTGGAG-NEP) for detecting $k d r$-TGT was labelled with NEP (Figure 2). The wild-type and mutated $k d r$ genotypes, TTG, TTT and TGT, were detected using a Roche Lightcycler480 via the FAM channel (465 nm-510 nm), the VIC/HEX channel (533 nm-580 nm) and the CY5 channel (618 nm-660 nm), respectively. The $10 \mu \mathrm{L}$ PCR reactions contained $1 \mu \mathrm{L}$ of genomic template, $5 \mu \mathrm{L}$ of $2 \times$ reaction master mix which contains $2 \times$ reaction buffer, dNTPs, Taq DNA polymerase, $\mathrm{Mg}^{2+}$ and $\mathrm{RNaseH}, 0.5 \mu \mathrm{L}$ of $10 \mu \mathrm{M}$ each primer, $0.4 \mu \mathrm{L}$ of $10 \mu \mathrm{M}$ each probe, and distilled water to $10 \mu \mathrm{L}$. PCR amplification was performed on a Roche Lightcycler480, using the following conditions: 5 minutes at $95^{\circ} \mathrm{C}$, followed by 40 cycles of $95^{\circ} \mathrm{C}$ for 15 seconds and $60^{\circ} \mathrm{C}$ for 30 seconds. The changes in FAM, VIC/HEX, and CY5 fluorescence were monitored in real time by acquiring the signal at $60^{\circ} \mathrm{C}$ for each cycle.

\section{Analysing the accuracy of AllGlo-qPCR compared to AS-PCR and TaqMan-qPCR}

To verify the accuracy of the AllGlo-qPCR method, the $k d r$ genotypes of both the field (108) and laboratory (50) samples were simultaneously detected using the AllGloqPCR method and two previously established approaches, AS-PCR and TaqMan-qPCR.

\section{Comparative analysis of AllGlo-qPCR, AS-PCR and TaqMan-qPCR}

The special equipment required, protocol run time, number of steps, primers/probes required, number of reaction tubes and the average cost per sample (excluding the cost of equipment and machine maintenance) were compared between AllGlo-qPCR, AS-PCR, TaqMan-qPCR and DNA sequencing.

\section{Detecting $k d r$ mutation frequencies in samples collected from central China}

The $k d r$ mutation frequency was investigated in samples from the other provinces in central China, including the

TTTCTTCATTTACTCCTGCCACTCCGTGTGTTTAGACAATGTGGATAGATTTCCCGACGGCGATCTGCCCAGATGGAATTTCACGGACT CTCCTTCATGATCGTGTTTCGCGTGCTGTGCGGGGAGTGGATCGAATCAATGTGGGACTGTATGCTAGTCGGGGATGTGTCATGCATC CCATTCTTCTTAGCCACTGTGGTAATIGGAAACITGGTGGTGAGTAACTGCAGGACGACAGGTCAGCAÇTTGCTCCGATTCTAATAAG Primer-F
ACGCTTTGGTTIAGgTACTTAATCTTTTCTTAGCTTTGCTTTTGTCCAATTTCGGATCATCATCGCTCTCTGCACCAACGGCGGATAAC GAGACGAATAAAATCGCTGAAGCGTTCAACCGAATTTCCCGCTTCTCTAACTGGATCAAAATGAACGTAGCAAATGCGCTAAAGTTTTT

Figure 2 Schematic representation of the primers and probes used to detect $k d r$ mutations in An. sinensis. Arrows represent the forward primer (left) and the reverse primer (right). The box represents the location where the probe used to detect wild-type (red) and mutated genotypes anneals. 
Henan, Hubei, Zhejiang and Shandong Provinces, using the AllGlo-qPCR method established above.

\section{Results}

Predominant kdr mutations in An. sinensis

Multiple mutated $k d r$ genotypes were identified via sequencing of 108 wild and 50 laboratory An. sinensis mosquitoes. All of the 50 laboratory samples were homozygous for the wild-type sequence (TTG/TTG). By contrast, $5 k d r$ genotypes were detected in the wild samples, including the dominant homozygotic genotype TTT/TTT in 61 mosquitoes, the heterozygotic genotype TTT/TGT in 30 mosquitoes, and low percentages of TTG/TTT, TTG/TGT and TGT/TGT genotypes (found in 3, 1 and 1 mosquitoes, respectively). In addition, 12 wild An. sinensis mosquitoes with the wild-type genotype (TTG/TTG) were identified.

\section{AllGlo-qPCR-based detection of $k d r$ mutations}

The AllGlo-qPCR assay detected the six known $k d r$ genotypes in An. sinensis and was 100\% consistent with sequencing results. Three probes were added simultaneously to a single reaction. A substantial increase in FAM fluorescence indicated a wild-type homozygote, and a substantial increase in $\mathrm{HEX} / \mathrm{VIC}$ or $\mathrm{CY} 5$ fluorescence indicated a homozygous mutant. An intermediate increase in any of the two signals indicated heterozygosity (Figure 3 ). The $k d r$ genotypes were scored with software, and the endpoint fluorescence intensities between any two dyes were plotted against each other on bi-directional scatter plots (Figure 4). All six of the known $k d r$ genotypes could be identified easily in comparison to the three bi-directed clusters.

\section{AllGlo-qPCR detected $k d r$ mutations with greater sensitivity and specificity than current methods}

The 158 samples with known $k d r$ genotypes were tested by AS-PCR, TaqMan-MGB and AllGlo-qPCR methods. A comparison of the results showed that TaqMan-qPCR and AllGlo-qPCR were more sensitive and specific than AS-PCR, as both methods demonstrated 100\% sensitivity with only one exception (discrimination of TTT/TTT) and $100 \%$ specificity with only one exception (discrimination of TTT/TGT) out of the six total $k d r$ genotypes. However, the AS-PCR method generated a relatively higher level of incorrect results, leading to lower sensitivity and specificity (Table 1). In general, the AllGlo-qPCR method was most accurate (98.73\%), followed by TaqMan-MGB (96.84\%) and AS-PCR (88.61\%) when compared to nucleotide sequencing (Table 1).

\section{AllGlo-qPCR is easier to use with moderate cost}

The equipment required, ease of use and the cost of four available methods for detecting $k d r$ mutations in $A n$. sinensis were summarized (Table 2). More specialized equipment and more steps are needed for sequencing and
AS-PCR, whereas only a real-time PCR machine and one experimental step are required for the other two methods. In addition, the TaqMan-MGB and AllGlo-qPCR methods do not entail the use of hazardous chemicals, such as ethidium bromide solution, and the run time (less than $2 \mathrm{~h}$ ) is much shorter than sequencing and AS-PCR. Sequencing was the most expensive technique, and AS-PCR was the least expensive. AllGlo-qPCR was less expensive than TaqMan-MGB, as only a single reaction tube was needed rather than two.

\section{High $k d r$ mutation frequencies were detected in mosquitoes collected from central China}

The $k d r$ mutation frequencies in An. sinensis samples collected from central China (Henan, Hubei, Zhejiang, Shandong and Jiangsu) were detected using the established AllGlo-qPCR method (Figure 1, Table 3). Mosquitoes from most of the provinces in central China exhibited high $k d r$ mutation frequencies (from $86.57 \%$ in Jiangsu to $97.71 \%$ in Zhejiang), with the exception of Hubei, where the mutation frequency was relatively low (45.38\%). Overall, 321 out of 337 individual An. sinensis mosquitoes (95.25\%) carried at least one copy of the TTT mutated $k d r$ allele.

\section{Discussion}

$K d r$ mutations are strongly associated with resistance to insecticides, especially pyrethroids and DDTs, in many malaria vectors. Previous studies have focused on screening for $k d r$ mutations in Anopheles mosquitoes. Currently, a number of assays are available for genotyping $k d r$ alleles, including AS-PCR [13], heated oligonucleotide ligation assay (HOLA), sequence-specific oligonucleotide probe enzyme-linked immunosorbent assay (SSOP-ELISA) [16], PCR-Dot, TaqMan probebased analyses, high-resolution melt (HRM) analysis $[17,18]$, rPASA, fluorescence resonance energy transfer/ melting curve analysis (FRET/MCA) [19], PCR extension with fluorescence [20], allele-specific loop-mediated isothermal amplification (AS-LAMP) [21], and more. ASPCR is the method that is most widely used in countries that are endemic for malaria, most likely due to its relatively low cost (in terms of the equipment needed and cost per run). However, the reliability of this technique is easily affected by the accuracy of primer design, optimization of the reaction system and the difficulty in distinguishing ambiguous bands, which limits its application. In recent years, more scientists have turned to real-time PCR-based assays to detect $k d r$ mutations, due to their ease of use and higher reliability $[17,22]$.

This report is the first to describe the development of an AllGlo-qPCR assay for detecting $k d r$ mutations in Anopheles mosquitoes. Unlike traditional design realtime probes such as TaqMan probes, which have a 


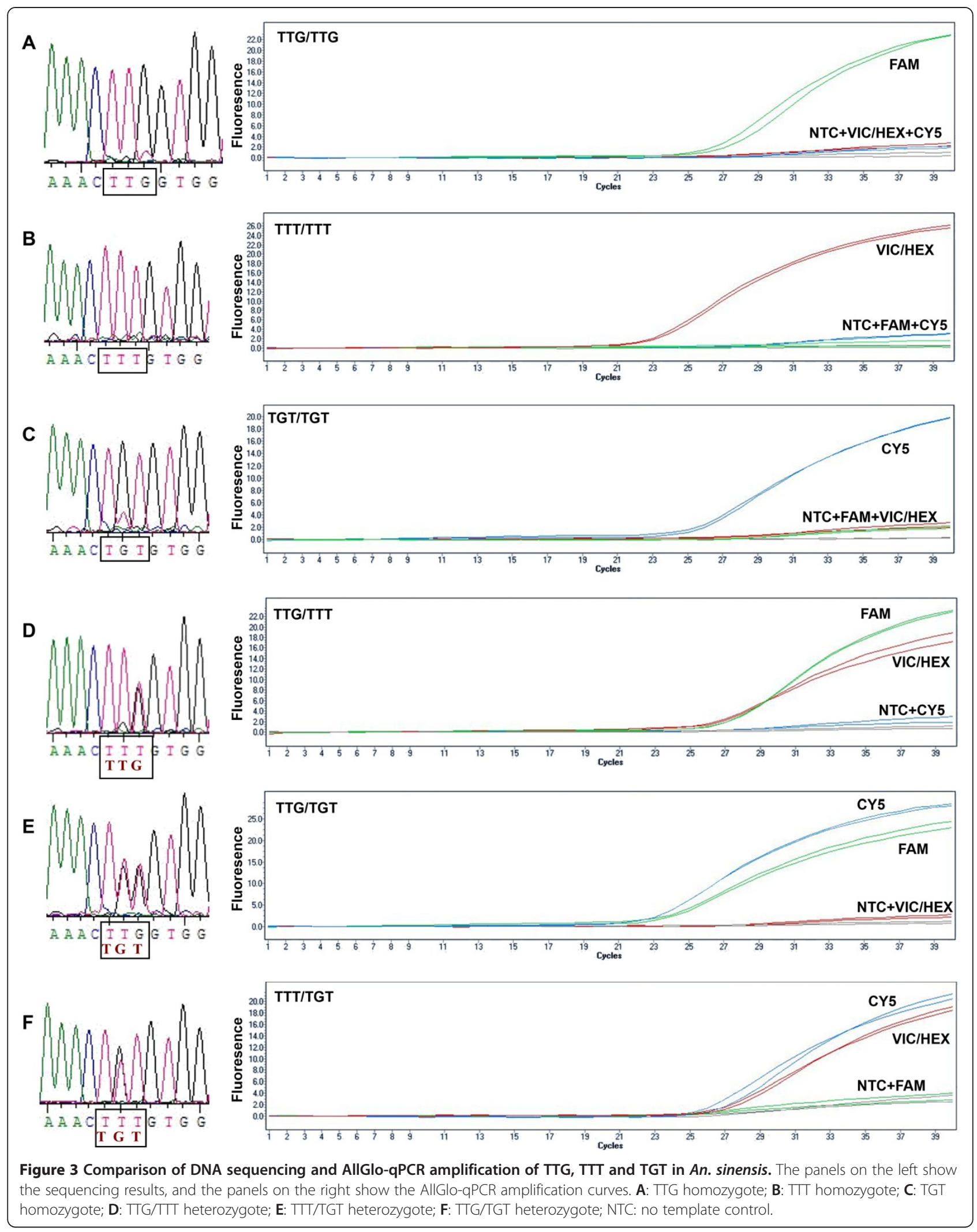




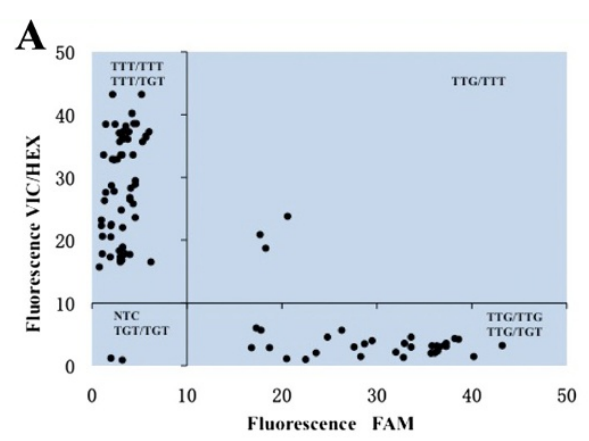

B

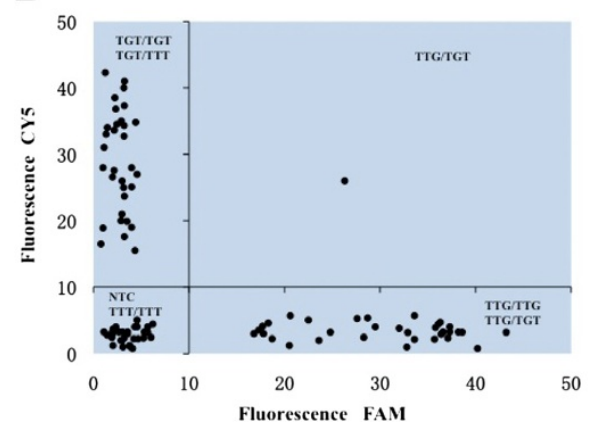

C

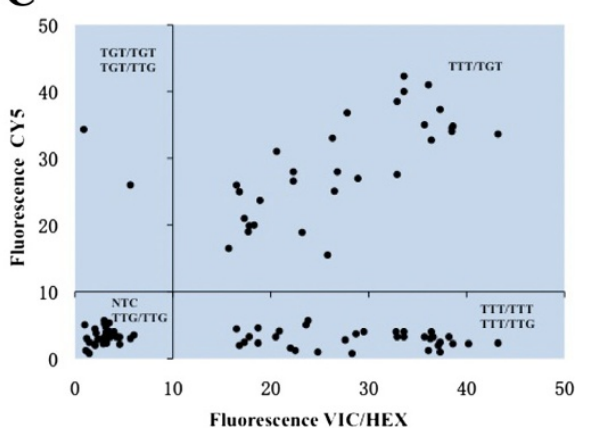

Figure 4 Scatter plot analysis of $\boldsymbol{k d r}$ mutations in An. sinensis detected by AllGlo-qPCR. Endpoint fluorescence intensities between (A) FAM and VIC/HEX; (B) FAM and CY5; (C) VIC/HEX and CY5. PCR was performed in 96-well plates. The FAM, VIC/HEX and CY5 fluorescence intensities were measured and corrected for background and then plotted in a bi-directional scatter plot using Microsoft Excel. NTC: no template control.

fluorophore at the 5 ' end and a nonfluorescent quencher at the 3' end, AllGlo probes have two identical reporter dyes that normally quench themselves. Upon hybridization with the target sequence, the labelled oligo becomes stretched and cleaved, leading to separation of the two reporter dyes and consequent fluorescence. Therefore, these probes offer much higher sensitivity than traditional TaqMan probes. This general observation is supported by the results from this study, which show that the AllGlo-qPCR method has greater sensitivity than the AS-PCR and TaqMan-MGB methods. Out of the 158 samples tested, the AllGlo-qPCR method generated only two incorrect genotyping results, which might have been due to poor DNA extraction and reaction conditions. Another advantage of AllGlo-qPCR is that it is less expensive than TaqMan-MGB. While the $k d r$ wild-type genotype and both mutations are detected in a single reaction tube in AllGlo-qPCR, two independent reaction tubes are required for the detection of these three $k d r$ alleles by TaqMan-qPCR. The use of fewer probes and reaction reagents and a simpler probe design contribute to the reduced cost of AllGlo-qPCR (Table 2). In addition, the clustering of samples in scatter plots leads to simple and high-throughput genotype scoring for detecting $k d r$ mutations. Therefore, use of the AllGlo-qPCR method should be considered in areas of malaria transmission to screen for $k d r$ mutations in $A n$. sinensis and other malaria vectors, as long as resources exist for purchasing and maintaining a real-time PCR machine.

The $k d r$ mutations were successfully detected by AllGloqPCR in An. sinensis samples collected from the other four provinces in central China, suggesting the wild An. sinensis mosquitoes in these regions share similar $k d r$ mutations as those in Jiangsu Province. The predominant $k d r$ allele detected was L1014 F (TTT), with a small percentage of L1014C (TGT) alleles, which is consistent with previous studies of $A n$. sinensis and other mosquito species, e.g., Culex pipiens pallens [7,23], indicating a similar genetic outcome under selective pressure from insecticide treatment. The high frequency of $k d r$ mutation (more than $87 \%$ ) observed in this study from samples collected in central China is consistent with the other studies of $A n$. sinensis $[24,25]$. A high level of resistance to insecticides (mainly the pyrethroids) has been reported in wild $A n$. sinensis in central China, and, according to previous studies, most of the regions with high insecticide resistance levels have high $k d r$ mutation frequencies $[7,24,26]$. However, an exception to this rule was found in the Hubei province, which has a high percentage of resistance in the wild An. sinensis population, though a relatively low frequency of $k d r$ mutation (45.38\%) was detected. In 2011, a higher frequency of $k d r$ mutation ( $94.8 \%$ of 122 wild samples) was detected in Wuxue, Hubei, an area of where many mosquitoes exhibit 
Table 1 Comparison of $k d r$ mutations in An. sinensis detected by AS-PCR, TaqMan-qPCR and AllGlo-qPCR versus direct sequencing

\begin{tabular}{|c|c|c|c|c|c|c|c|c|c|c|}
\hline \multirow[t]{2}{*}{ Genotype } & \multicolumn{4}{|c|}{ Detection assay* } & \multicolumn{3}{|c|}{ Sensitivity ( $95 \%$ confidence level) } & \multicolumn{3}{|c|}{ Specificity ( $95 \%$ confidence level) } \\
\hline & Sequencing & AS-PCR & TaqMan- qPCR & AllGlo-qPCR & AS-PCR & TaqMan- qPCR & AllGlo-qPCR & AS-PCR & TaqMan- qPCR & AllGlo-qPCR \\
\hline TTG/TTG & 62 & 59 & 62 & 62 & $95 \%(87 \%, 98 \%)$ & $100 \%(94 \%, 100 \%)$ & $100 \%(94 \%, 100 \%)$ & $100 \%(96 \%, 100 \%)$ & $100 \%(96 \%, 100 \%)$ & $100 \%(96 \%, 100 \%)$ \\
\hline$\pi \pi / \pi \pi$ & 61 & $60^{\mathrm{a}}$ & 56 & 59 & $89 \%(78 \%, 94 \%)$ & $92 \%(82 \%, 96 \%)$ & $97 \%(89 \%, 99 \%)$ & $94 \%(87 \%, 97 \%)$ & $100 \%(96 \%, 100 \%)$ & $100 \%(96 \%, 100 \%)$ \\
\hline TGT/TGT & 1 & $2^{b}$ & 1 & 1 & $100 \%(21 \%, 100 \%)$ & $100 \%(21 \%, 100 \%)$ & $100 \%(21 \%, 100 \%)$ & $99 \%(96 \%, 99.9 \%)$ & $100 \%(98 \%, 100 \%)$ & $100 \%(98 \%, 100 \%)$ \\
\hline$T \mathrm{TG} / \mathrm{TT}$ & 3 & $5^{c}$ & 3 & 3 & $67 \%(21 \%, 94 \%)$ & $100 \%(44 \%, 100 \%)$ & $100 \%(44 \%, 100 \%)$ & $98 \%(94 \%, 99 \%)$ & $100 \%(98 \%, 100 \%)$ & $100 \%(98 \%, 100 \%)$ \\
\hline TTG/TGT & 1 & $2^{d}$ & 1 & 1 & $100 \%(21 \%, 100 \%)$ & $100 \%(21 \%, 100 \%)$ & $100 \%(21 \%, 100 \%)$ & $99 \%(96 \%, 99.9 \%)$ & $100 \%(98 \%, 100 \%)$ & $100 \%(98 \%, 100 \%)$ \\
\hline TT/TGT & 30 & $30^{\mathrm{e}}$ & $35^{f}$ & $32^{9}$ & $77 \%(59 \%, 88 \%)$ & $100 \%(89 \%, 100 \%)$ & $100 \%(89 \%, 100 \%)$ & $95 \%(89 \%, 97 \%)$ & $96 \%(91 \%, 98 \%)$ & $98 \%(94 \%, 99.6 \%)$ \\
\hline Total & 158 & 158 & 158 & 158 & & & & & & \\
\hline Accuracy** & - & $88.61 \%$ & $96.84 \%$ & $98.73 \%$ & & & & & & \\
\hline
\end{tabular}

*:The superscript letter indicates an incorrect result generated by AS-PCR, TaqMan-qPCR or AllGlo-qPCR, compared to standard sequencing, as follows: a: 6 TाT/TGT; b: 1 TाT/TGT; c: 3 TTG/TTG; d: 1 TTG/TT; e: 7 TाT/

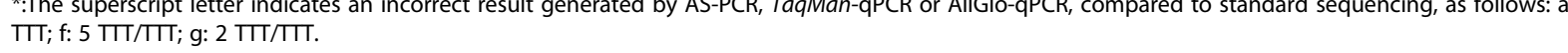

**:The accuracy refers to the percentage of correct results generated by AS-PCR, TaqMan-qPCR or AllGlo-qPCR out of 158 known kdr genotype samples. 
Table 2 Comparison of four kdr genotyping assays based on the need for specialized equipment, cost, safety, simplicity and speed

\begin{tabular}{|c|c|c|c|c|c|c|c|}
\hline Method & Equipment required & $\begin{array}{l}\text { Hazardous } \\
\text { chemicals }\end{array}$ & $\begin{array}{l}\text { Protocol } \\
\text { run time }\end{array}$ & $\begin{array}{l}\text { No. of } \\
\text { steps }\end{array}$ & $\begin{array}{l}\text { Primers/probe } \\
\text { required }\end{array}$ & $\begin{array}{l}\text { No. of tubes required } \\
\text { per sample }\end{array}$ & $\begin{array}{l}\text { Cost per } \\
\text { run* }\end{array}$ \\
\hline \multirow[t]{3}{*}{ Sequencing } & PCR thermocycler & Ethidium bromide & $6 \mathrm{~h}$ & 2 & 2 PCR primers & 1 & $\$ 4.20$ \\
\hline & Gel electrophoresis and imaging equipment & & & & & & \\
\hline & Nucleic acid sequencing machine & & & & & & \\
\hline \multirow[t]{2}{*}{ AS-PCR } & PCR thermocycler & Ethidium bromide & $4 \mathrm{~h} 30 \mathrm{~min}$ & 2 & 5 PCR primers & 2 & $\$ 0.25$ \\
\hline & Gel electrophoresis and imaging equipment & & & & & & \\
\hline \multirow[t]{2}{*}{ TaqMan-qPCR } & Real-time PCR machine & - & $1 \mathrm{~h} 45 \mathrm{~min}$ & 1 & $2 \mathrm{PCR}$ primers & 2 & $\$ 0.70$ \\
\hline & & & & & 3 fluorescently labelled probes & & \\
\hline \multirow[t]{2}{*}{ AllGlo-qPCR } & Real-time PCR machine & - & $1 \mathrm{~h} 45 \mathrm{~min}$ & 1 & $2 \mathrm{PCR}$ primers & 1 & $\$ 0.45$ \\
\hline & & & & & 3 fluorescently labelled probes & & \\
\hline
\end{tabular}

*:Refers to the average cost to run one sample; the cost of the equipment was not included. 
Table $3 \mathrm{Kdr}$ mutation frequencies in An. sinensis samples from central China, as detected by AllGlo-qPCR

\begin{tabular}{|c|c|c|c|c|c|c|c|c|}
\hline \multirow[t]{2}{*}{ Field site } & \multirow[t]{2}{*}{ Sample size } & \multicolumn{6}{|c|}{ Genotype } & \multirow{2}{*}{$\begin{array}{l}\text { Mutation } \\
\text { frequency }\end{array}$} \\
\hline & & TTG/TTG & TTT/TTT & TGT/TGT & TTG/TTT & TTG/TGT & TTT/TGT & \\
\hline Henan & 42 & 1 & 18 & 7 & 6 & 0 & 10 & $90.48 \%$ \\
\hline Hubei & 48 & 22 & 9 & 3 & 7 & 0 & 7 & $45.38 \%$ \\
\hline Zhejiang & 131 & 1 & 105 & 3 & 4 & 0 & 18 & $97.71 \%$ \\
\hline Shandong & 48 & 4 & 28 & 1 & 1 & 0 & 14 & $90.63 \%$ \\
\hline Jiangsu & 108 & 12 & 61 & 1 & 3 & 1 & 30 & $86.57 \%$ \\
\hline Total & 377 & 40 & 221 & 15 & 21 & 1 & 79 & $86.47 \%$ \\
\hline
\end{tabular}

deltamethrin resistance [7], suggesting that the frequency of $k d r$ mutation differs significantly according to the mosquito collection site, even in areas with similar geographical characteristics [27]. Another possible explanation is that other factors could also be involved in the insecticide resistance. For example, insecticide resistance levels were recently found to continue to increase during the pyrethroid selection process even when the $k d r$ mutation frequency reaches a maximum (100\%) in a wild An. sinensis population (unpublished data). These observations are consistent with a recent study conducted in Anhui (Figure 1), where the authors concluded that metabolic detoxification, and not the L1014 $k d r$ mutation, may be the dominant mechanism of insecticide resistance in An. sinensis in this region [28], suggesting a complex mechanism for insecticide resistance in An. sinensis. However, identifying the mechanism of insecticide resistance in An. sinensis is outside of the scope of the present study, as the main purpose was to establish the novel AllGlo-qPCR method for detecting $k d r$ mutations.

\section{Conclusion}

This report is the first to describe a high-throughput AllGlo-qPCR assay that can be used to detect $k d r$ mutations in Anopheles mosquitoes. Compared to two other previously reported methods, the AllGlo-qPCR method delivers the greatest specificity and sensitivity at a reasonable cost per run. This assay could be widely used to screen for $k d r$ mutations in An. sinensis in central China, and it has the potential to be used for other mosquito species in regions of malaria transmission.

\section{Competing interests}

The authors declare that they have no competing interests.

\begin{abstract}
Authors' contributions
$L B, G D Z$, JC and QG conceived and designed the study; LB performed the experiments; LB, GDZ and MHZ analysed the data; GDZ, HYZ, JXT, JLL, SX, LNY, GQH, YBW and HWZ contributed to sample collection and species identification; GDZ and LB drafted the manuscript; and SBW, JC and QG revised the manuscript. All of the authors read and approved the final manuscript.
\end{abstract}

\section{Acknowledgements}

The authors would like to thank Luhe, Sihong and the Yixing County Center for Disease Control and Prevention (CDC) in Jiangsu for assistance with the mosquito collection. This work was supported by the Natural Science
Foundation of Jiangsu Province (BK20141106 and BK2012106), Jiangsu Province's Construction Project (BM2009902), Jiangsu Province's Medical High Tech Platform (ZX201108) and the National S \& T Major Programme (2012ZX10004220). The funders had no role in study design, data collection and analysis, the decision to publish, or preparation of the manuscript.

\section{Author details}

${ }^{1}$ Key Laboratory of Parasitic Disease Control and Prevention (Ministry of Health), and Jiangsu Provincial Key Laboratory of Parasite Molecular Biology, Jiangsu Institute of Parasitic Diseases, Wuxi, Jiangsu Province, People's Republic of China. ${ }^{2}$ Institute of Plant Physiology \& Ecology, Shanghai Institute for Biological Sciences, Chinese Academy of Sciences, Shanghai, People's Republic of China. ${ }^{3}$ Department of Parasitology, Medical College of Soochow University, Suzhou 215123, People's Republic of China. ${ }^{4}$ Zhejiang Center for Disease Control and Prevention, Hangzhou, People's Republic of China. ${ }^{5}$ Hubei Center for Disease Control and Prevention, Wuhan, People's Republic of China. 'Shandong Institute of Parasitic Diseases, Jining, People's Republic of China. ${ }^{7}$ Henan Center for Disease Control and Prevention, Zhengzhou, People's Republic of China. ${ }^{8}$ Public Health Research Center, Jiangnan University, Wuxi, People's Republic of China.

Received: 16 July 2014 Accepted: 20 September 2014 Published: 23 September 2014

\section{References}

1. Sinka ME, Bangs MJ, Manguin S, Chareonviriyaphap T, Patil AP, Temperley WH, Gething PW, Elyazar IR, Kabaria CW, Harbach RE, Hay SI: The dominant Anopheles vectors of human malaria in the Asia-Pacific region: occurrence data, distribution maps and bionomic precis. Parasit Vectors 2011, 4:89.

2. Zhou SS, Zhang SS, Wang JJ, Zheng X, Huang F, Li WD, Xu X, Zhang HW: Spatial correlation between malaria cases and water-bodies in Anopheles sinensis dominated areas of Huang-Huai plain. China Parasit Vectors 2012, 5:106.

3. Zhu G, Xia H, Zhou H, Li J, Lu F, Liu Y, Cao J, Gao Q, Sattabongkot J: Susceptibility of Anopheles sinensis to Plasmodium vivax in malarial outbreak areas of central China. Parasit Vectors 2013, 6:176.

4. Pan JY, Zhou SS, Zheng X, Huang F, Wang DQ, Shen YZ, Su YP, Zhou GC, Liu F, Jiang JJ: Vector capacity of Anopheles sinensis in malaria outbreak areas of central China. Parasit Vectors 2012, 5:136.

5. The malERA Consultative Group on Vector Control: A research agenda for malaria eradication: vector control. PLOS Med 2011, 8:e1000401.

6. Ranson H, N'Guessan R, Lines J, Moiroux N, Nkuni Z, Corbel V: Pyrethroid resistance in African anopheline mosquitoes: what are the implications for malaria control? Trends Parasitol 2011, 27:91-98.

7. Zhong D, Chang X, Zhou G, He Z, Fu F, Yan Z, Zhu G, Xu T, Bonizzoni M, Wang M-H, Cui L, Zheng B, Chen B, Yan G: Relationship between knockdown resistance, metabolic detoxification and organismal resistance to pyrethroids in Anopheles sinensis. PLoS One 2013, 8:e55475.

8. Wang DQ, Xia ZG, Zhou SS, Zhou XN, Wang RB, Zhang QF: A potential threat to malaria elimination: extensive deltamethrin and DDT resistance to Anopheles sinensis from the malaria-endemic areas in China. Malar J 2013, 12:164.

9. Oxborough RM, Kitau J, Jones R, Feston E, Matowo J, Mosha FW, Rowland MW: Long-lasting control of Anopheles arabiensis by a single spray 
application of micro-encapsulated pirimiphos-methyl (Actellic(R) 300 CS). Malar J 2014, 13:37.

10. Hemingway J, Hawkes NJ, McCarroll L, Ranson H: The molecular basis of insecticide resistance in mosquitoes. Insect Biochem Mol Biol 2004, 34:653-665.

11. Tan WL, Wang ZM, Li CX, Chu HL, XU Y, Dong YD, Wang ZC, Chen DY, Liu H, Liu DP, Liu N, Sun J, Zhao T: First report on co-occurrence knockdown resistance mutations and susceptibility to beta-cypermethrin in Anopheles sinensis from Jiangsu Province. China PLoS One 2012, 7:e29242.

12. Kang S, Jung J, Lee $\mathrm{S}$, Hwang H, Kim W: The polymorphism and the geographical distribution of the knockdown resistance (kdr) of Anopheles sinensis in the Republic of Korea. Malar J 2012, 11:151.

13. Wu S, Ma EJ, Liu Q, Lu Q, Yang FT, Hong JF, Ye MQ, Yu JF: Establishment of AS-PCR method for detecting the knockdown resistance of Anopheles sinensis. Zhongguo Ren Shou Gong Huan Bing Xue Bao 2011, 27:1008-1010.

14. Bai L, Zhu GD, Tang JX, Zhang C, Liu YB, Li JL, Cao J, Gao Q: Study on TaqMan-MGB real-time fluorescence quantitative PCR to detect gene mutation of kdr from Anopheles sinensis. Zhongguo Xue Xi Chong Bing Fang Zhi Za Zhi 2013, 25:167-171. 176.

15. Gao Q, Beebe NW, Cooper RD: Molecular identification of the malaria vectors Anopheles anthropophagus and Anopheles sinensis (Diptera: (ulicidae) in central China using polymerase chain reaction and appraisal of their position within the Hyrcanus group. J Med Entomol 2004, 41:5-11.

16. Kulkarni MA, Rowland M, Alifrangis M, Mosha FW, Matowo J, Malima R, Peter J, Kweka E, Lyimo I, Magesa S, Salanti A, Rau ME, Drakeley C: Occurrence of the leucine-to-phenylalanine knockdown resistance (kdr) mutation in Anopheles arabiensis populations in Tanzania, detected by a simplified high-throughput SSOP-ELISA method. Malar J 2006, 5:56.

17. Bass C, Nikou D, Donnelly MJ, Williamson MS, Ranson H, Ball A, Vontas J, Field LM: Detection of knockdown resistance $(\mathrm{kdr})$ mutations in Anopheles gambiae: a comparison of two new high-throughput assays with existing methods. Malar J 2007, 6:111.

18. Lynd A, Ranson H, McCall PJ, Randle NP, Black WCT, Walker ED, Donnelly MJ: A simplified high-throughput method for pyrethroid knock-down resistance (kdr) detection in Anopheles gambiae. Malar J 2005, 4:16.

19. Verhaeghen K, Van Bortel W, Roelants P, Backeljau T, Coosemans M: Detection of the East and West African kdr mutation in Anopheles gambiae and Anopheles arabiensis from Uganda using a new assay based on FRET/Melt Curve analysis. Malar J 2006, 5:16.

20. Tripet F, Wright J, Lanzaro G: A new high-performance PCR diagnostic for the detection of pyrethroid knockdown resistance kdr in Anopheles gambiae. Am J Trop Med Hyg 2006, 74:658-662.

21. Badolo A, Okado K, Guelbeogo WM, Aonuma H, Bando H, Fukumoto S, Sagnon N, Kanuka H: Development of an allele-specific, loop-mediated, isothermal amplification method (AS-LAMP) to detect the L1014F kdr-w mutation in Anopheles gambiae s. I. Malar J 2012, 11:227.

22. Choi KS, Spillings BL, Coetzee M, Hunt RH, Koekemoer LL: A comparison of DNA sequencing and the hydrolysis probe analysis (TaqMan assay) for knockdown resistance (kdr) mutations in Anopheles gambiae from the Republic of the Congo. Malar J 2010, 9:278.

23. Chen L, Zhong D, Zhang D, Shi L, Zhou G, Gong M, Zhou H, Sun Y, Ma L, He J, Hong S, Zhou D, Xiong C, Zou P, Zhu C, Yan G: Molecular ecology of pyrethroid knockdown resistance in Culex pipiens pallens mosquitoes. PLoS One 2010, 5:e11681.

24. Chang XL, Xue YQ, Zhang AD, Zhu GD, Fang Q: Deltamethrin resistance, metabolic detoxification enzyme and kdr mutation in Anopheles sinensis in region along Huaihe River in Anhui Province. Zhongguo Xue Xi Chong Bing Fang Zhi Za Zhi 2013, 25:263-267.

25. Xu T, Zhong D, Tang L, Chang X, Fu F, Yan G, Zheng B: Anopheles sinensis mosquito insecticide resistance: comparison of three mosquito sample collection and preparation methods and mosquito age in resistance measurements. Parasit Vectors 2014, 7:54.

26. Zhu G, Zhong D, Cao J, Zhou H, Li J, Liu Y, Bai L, Xu S, Wang MH, Zhou G, Chang X, Gao Q, Yan G: Transcriptome profiling of pyrethroid resistant and susceptible mosquitoes in the malaria vector, Anopheles sinensis. BMC Genomics 2014, 15:448.

27. Hyunwoo Kima JHB, Lee W-J, Lee SH: Frequency detection of pyrethroid resistance allele in Anopheles sinensis populations by real-time PCR amplification of specific allele (rtPASA). Pestic Biochem Phys 2007, 87:8.
28. Chang X, Zhong D, Fang Q, Hartsel J, Zhou G, Shi L, Fang F, Zhu C, Yan G: Multiple Resistances and Complex Mechanisms of Anopheles sinensis Mosquito: A Major Obstacle to Mosquito-Borne Diseases Control and Elimination in China. PLoS Negl Trop Dis 2014, 8:e2889.

doi:10.1186/1475-2875-13-379

Cite this article as: Bai et al:: Development and application of an AllGlo probe-based QPCR assay for detecting knockdown resistance ( $k d r)$ mutations in Anopheles sinensis. Malaria Journal 2014 13:379.

\section{Submit your next manuscript to BioMed Central and take full advantage of:}

- Convenient online submission

- Thorough peer review

- No space constraints or color figure charges

- Immediate publication on acceptance

- Inclusion in PubMed, CAS, Scopus and Google Scholar

- Research which is freely available for redistribution

Submit your manuscript at www.biomedcentral.com/submit
C Biomed Central 